\title{
Ziele von Informationssystemen
}

\author{
Christian Eller • René Riedl
}

Online publiziert: 11. April 2016

(C) The Author(s) 2016. This article is available at SpringerLink with Open Access

Zusammenfassung Voraussetzung für einen effektiven und effizienten Einsatz und Betrieb von Informationssystemen ist eine systematische Zielplanung. Je nach Einsatzgebiet gibt es verschiedenste Sachziele. Darüber hinaus gibt es aber allgemeine Formalziele, die für den erfolgreichen Einsatz und Betrieb von Informationssystemen erfolgsentscheidend, jedoch nur unzureichend erforscht sind. Zweck dieses Beitrags ist es, die Zielforschung voranzutreiben. Dazu werden durch Analyse der Fachliteratur strategische Formalziele von Informationssystemen identifiziert und ein Zielsystem entwickelt. Weiter wird auf der Basis einer explorativen Befragung von IT-Verantwortlichen die aktuelle und zukünftig erwartete Bedeutung von Formalzielen in der Praxis erhoben.

Als die konzeptionell bedeutsamsten strategischen Formalziele wurden Wirksamkeit und Wirtschaftlichkeit identifiziert. Die Wirksamkeit hängt von der Funktionalität und der tatsächlichen Nutzung eines Systems ab, welche wiederum von der Nutzerzufriedenheit beeinflusst wird. Bedeutsame Ziele, die Nutzerzufriedenheit beeinflussen, sind System-, Informations- und Servicequalität. Die meisten der 44 identifizierten Formalziele konnten der Systemqualität zugeordnet werden. Nachhaltigkeit gilt als höchstes Ziel. Das entwickelte Zielsystem soll als Grundlage für

Zusätzliche Informationen sind in der Online-Version dieses Artikels enthalten: 10.1365/s40702016-0219-8.

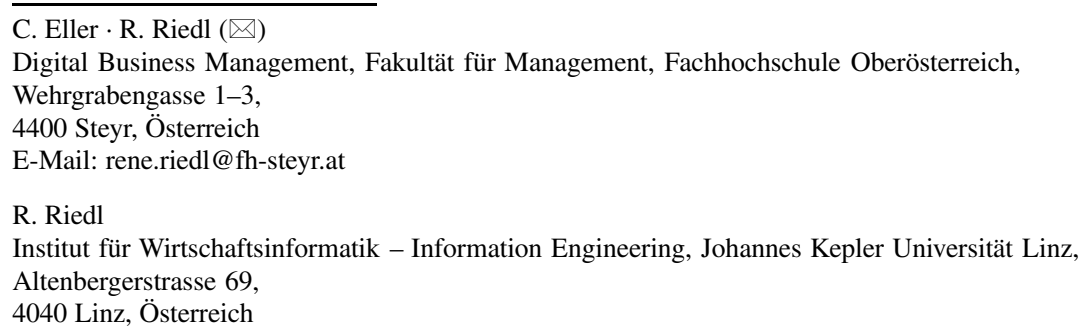


künftige Forschungsarbeiten dienen und kann in Unternehmen für die strategische Zielplanung herangezogen werden; dazu werden Handlungsempfehlungen gegeben.

Die Ergebnisse der Befragungsstudie zeigen, dass in Organisationen nur vereinzelt eine systematische Zielplanung durchgeführt wird. Die Befragten geben zwar an, viele der beschriebenen Formalziele zu verfolgen, doch die wenigsten davon werden tatsächlich gemessen. Zudem gibt nur eine Minderheit der Befragten an, Zielbeziehungen bei der Planung zu berücksichtigen. Verfügbarkeit, Nutzerzufriedenheit und Sicherheit sind die wichtigsten Formalziele und diese werden auch am häufigsten gemessen. Die Befragten geben zudem an, dass die Bedeutung der Ziele Entscheidungsunterstützung, Wirtschaftlichkeit, Nachhaltigkeit und Schlankheit in den nächsten fünf Jahren steigen wird.

Schlüsselwörter Informationsmanagement $\cdot$ IT-Controlling $\cdot$ IT-Planung $\cdot$ ITZiele $\cdot$ Strategisches IT-Management $\cdot$ Zielplanung $\cdot$ IT-Erfolg

\section{Information Systems Goals}

Abstract To run information systems (IS) effectively and efficiently, systematic planning of IS goals is needed. IS may serve different purposes. In addition to goals that result from a system's purpose, a number of critical goals related to IS quality aspects exist; yet, these goals are not well understood today. Thus, the objective of this article is to contribute to IS goal research. To this end, we analyzed the scientific literature to identify strategic IS goals and we developed a goal taxonomy. Moreover, based on an exploratory survey study of IT managers, we develop insight into the current and expected importance of goals in practice.

Conceptually, effectiveness und efficiency are the most important goals. Effectiveness depends on functionality and actual system usage, which, in turn, is affected by user satisfaction. The most important antecedents of user satisfaction are system quality, information quality, and service quality. Most of the 44 goals which we identified are related to system quality. Sustainability stands above all other goals. The goal taxonomy that we develop in this article may serve as a foundation for future research and may also be used in organizations for strategic planning purposes; recommendations for actions in pratice are given.

Results of the survey show that systematic goal planning hardly exists in organizations today. The respondents indicate that they pursue many of the goals examined in this study; yet, only a few of them are actually measured. Moroever, only a minority of the respondents indicate that they consider the relations between goals in their planning efforts. Availability, user satisfaction, and security are the most important goals and are measured most frequently. Also, respondents indicate that decision support, efficiency, sustainability, and plainness are goals with increasing importance in the next five years.

Keywords Information Management - IT Controlling · IT Planning · IT Goals · Strategic IT Management · Goal Planning · IT Success 


\section{Zielforschung vor 30 Jahren}

Bedeutsame Aufgaben des Informationsmanagements sind das Setzen von Zielen und das Messen der Zielerreichung als Grundvoraussetzung für die Planung, Überwachung und Steuerung der Informationsinfrastruktur einer Organisation. Diese Aufgaben sollten auf der strategischen, administrativen und operativen Ebene des Informationsmanagements realisiert sein. In der Wirtschaftsinformatik unterscheidet man üblicherweise zwischen Sach- und Formalzielen (Heinrich et al. 2011, vgl. dort den Abschn. „Ziele und Zielsystem“). Sachziele haben Zielinhalte, welche auf den Zweck eines Informationssystems ausgerichtet sind, während die Zielinhalte von Formalzielen die Qualität oder die Güte von Informationssystemen beschreiben.

Heinrich und Sterrer (1987) veröffentlichten vor rund drei Jahrzehnten die Ergebnisse einer empirischen Studie (Befragung von vorwiegend EDV-Leitern in zwölf österreichischen Unternehmen verschiedener Branchen) zu Formalzielen, die bei der Planung von Informationssystemen verfolgt werden. Konkret wurden zehn Formalziele (Akzeptanz, Aufgabenbezogenheit, Benutzerorientierung, Flexibilität, Integrationsfähigkeit, Koordinationsfähigkeit, Produktivität, Sicherheit, Wirtschaftlichkeit und Zuverlässigkeit) untersucht. Die Befunde der Studie zeigen, dass die befragten Unternehmen in den 1980er-Jahren kaum über eine systematische Zielplanung für Informationssysteme verfügten, auch wenn die Zweckmäßigkeit der Ziele von den Befragten damals bereits überwiegend bestätigt wurde.

Im vorliegenden Beitrag wird der aktuelle Stand der Zielforschung im Bereich der Informationssysteme beschrieben. In Anlehnung an Heinrich und Sterrer (1987) wird zudem über ausgewählte Befunde einer aktuellen explorativen Untersuchung berichtet, die Auskunft über den Status quo der Zielplanung in der Praxis gibt. Die Befunde der aktuellen Untersuchung zeigen, dass trotz der seit den 1980er-Jahren angestiegenen Verfügbarkeit von Forschungsergebnissen zu Zielen von Informationssystemen in weiten Teilen der Praxis nach wie vor keine systematische Zielplanung durchgeführt wird. Dies ist ein ernüchternder Befund, da seit Jahrzehnten die große Bedeutung der Zielplanung für den Erfolg des Informationsmanagements in Wissenschaft und Praxis bekannt und weithin unbestritten ist.

\section{Zielforschung heute}

\subsection{Strategische Formalziele}

Ziele beschreiben Zustände, die erreicht werden sollen. Man unterscheidet zwischen Sach- und Formalzielen. Nur Formalziele können unabhängig von der individuellen Unternehmenssituation analysiert werden (Heinrich et al. 2014, S. 139); sie sind deshalb Gegenstand dieses Beitrags sowie der empirischen Zielforschung im Allgemeinen.

Ziele können nach ihrer Wirkungsweise in strategisch, administrativ und operativ gruppiert werden (Heinrich et al. 2004, S. 738 f.). Im Gegensatz zu operativen Zielen zeichnen sich strategische Ziele durch folgende Merkmale aus: Betonung der Wichtigkeit (Relevanz), Beschränkung auf einige wesentliche Gesichtspunkte (Ver- 
einfachung) und Streben nach frühzeitigem Handeln (Proaktivität); administrative Ziele liegen zwischen operativen und strategischen. Aus den strategischen Zielen können administrative und operative Ziele abgeleitet werden. Der Fokus dieser Arbeit liegt auf strategischen Formalzielen. Diese Ziele sind in erster Linie für das Top-Management eines Unternehmens handlungsrelevant (z. B. Chief Information Officer).

Im folgenden Abschnitt wird die Frage beantwortet, welche Formalziele bei der Planung von betrieblichen Informationssystemen verfolgt werden können. Ausgehend von den zehn Zielen von Heinrich und Sterrer (1987) wurde die einschlägige Fachliteratur nach Formalzielen durchsucht, die heute als relevant erachtet werden. Das Ergebnis dieses Analyseprozesses ist eine Liste aktueller Formalziele von betrieblichen Informationssystemen (insgesamt 44 Ziele), welche als Basis für die explorative Studie diente (in die 21 der 44 Ziele aufgenommen wurden; die Selektion wurde von den Autoren durchgeführt und diente insbesondere dazu, ähnliche und teilweise sogar synonyme Begriffe zu bereinigen und die Dauer der Befragung in einem akzeptablen Ausmaß zu halten). Die Liste der Formalziele wurde zudem in ein Zielsystem überführt, um Abhängigkeiten zwischen den Zielen transparent zu machen.

\subsection{Literaturanalyse}

Ein Großteil der einschlägigen Fachliteratur stützt sich auf die Zielforschung der 1980er-Jahre. Nur wenige Autoren in der Wirtschaftsinformatik und InformationSystems-Disziplin (zur Unterscheidung der beiden Disziplinen siehe Heinrich et al. 2011, vgl. dort den Abschn. „Wirtschaftsinformatik versus Information Systems“) haben die Forschung auch in jüngerer Zeit weiter vorangetrieben (die Ergebnisse einer systematischen Literaturrecherche in den Datenbanken von ACM, IEEE und Springer sowie auf EBSCO HOST und in WEB OF SCIENCE werden auf Anfrage gerne zur Verfügung gestellt). Viele Autoren beschäftigten sich zudem mit dem Thema IT-Controlling, das per Definition Aussagen zu Zielen von Informationssystemen enthalten sollte. Überraschenderweise konnten in vielen Arbeiten zum IT-Controlling (vgl. z. B. die in Heinrich et al. 2011 im Abschn. „Controlling“ genannten Literaturquellen) meist keine Formalziele identifiziert werden, da die Werke typischerweise den Zielfindungsprozess beschreiben und/oder auf spezifische Sachziele eingehen (vgl. Ausnahmen wie z. B. Roithmayr 1988, der auf Basis einer empirische Studie Formalziele wie Sicherheits-, Effizienz-, Durchdringungs- und Anpassungsstreben identifizierte, sowie den Abschn. „IT-Controlling“ in Krcmar 2010 und den Abschn. „Management der Informationsverarbeitung“ in Mertens et al. 2012). Forschungsanstrengungen wurden auch im Bereich der Strategischen Informationssystemplanung (SISP) unternommen (siehe z. B. Teubner 2013), aber auch hier konnten nur wenige Hinweise auf Formalziele gefunden werden. Weitere Forschung, die insbesondere in Journalen der Information-Systems-Disziplin veröffentlicht ist, beschäftigte sich damit, den Erfolg von Informationssystemen messbar zu machen (z. B. DeLone und McLean 2003). Aus dieser Forschungsrichtung konnten etliche Formalziele von Informationssystemen abgeleitet werden. 


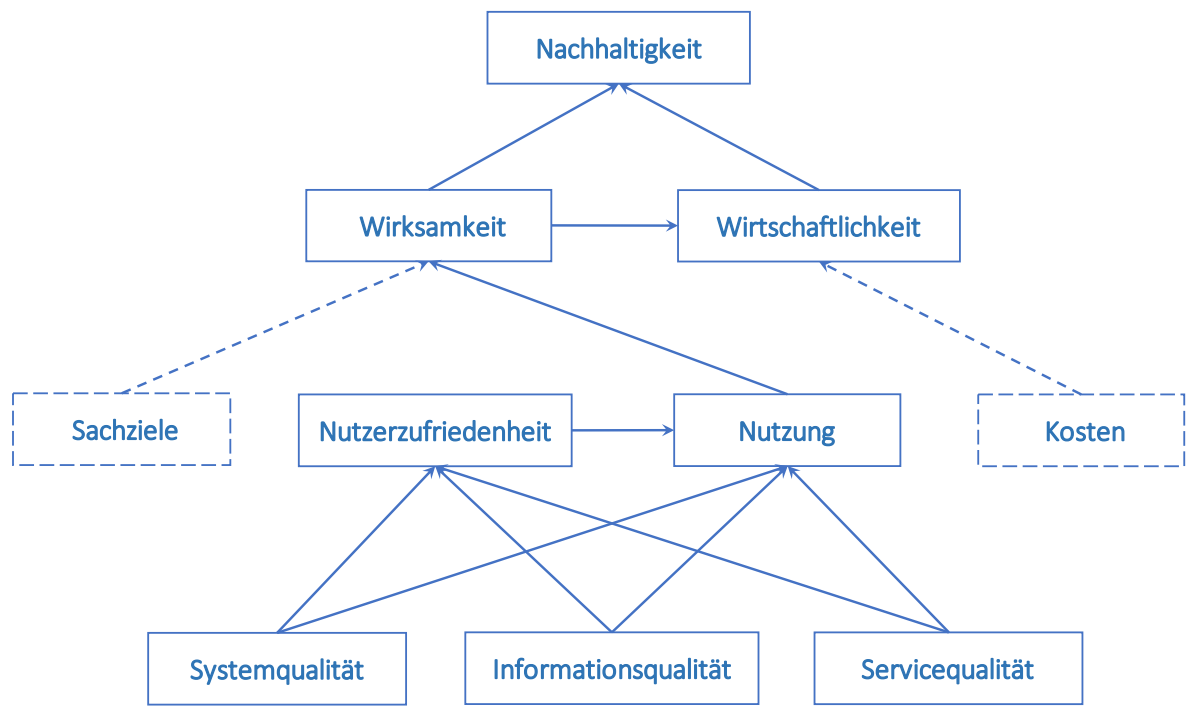

Abb. 1 Zielsystem strategischer Formalziele von Informationssystemen

\subsection{Entwicklung eines Zielsystems}

Um ein Zielsystem zu bilden, wurden die im Zuge der Literaturrecherche identifizierten Ziele definiert und in Beziehung zueinander gesetzt. Unabhängig von allen anderen Zielen haben sich aus der Analyse Wirksamkeit und Wirtschaftlichkeit als zentrale Ziele von Informationssystemen herauskristallisiert. Die Wirtschaftlichkeit wird zum einen von der Wirksamkeit bestimmt und zum anderen von den Kosten, die entstehen, um die gewünschte Wirksamkeit zu erzielen (siehe Abb. 1). Die Wirksamkeit wiederum hängt einerseits von den verfolgten Sachzielen ab, andererseits von der tatsächlichen Systemnutzung. Wie DeLone und McLean (2003) gezeigt haben, führen eine hohe Systemqualität, eine hohe Informationsqualität und eine entsprechende Servicequalität zu einer hohen Nutzerzufriedenheit und zur gewünschten Nutzung eines Systems. Über allen Zielen steht die Nachhaltigkeit. Es gilt Informationssysteme langfristig ökonomisch, ökologisch und sozial verträglich zu planen und einzusetzen, dies setzt Wirksamkeit und Wirtschaftlichkeit voraus.

Die meisten bei der Recherche identifizierten Ziele wurden der Systemqualität zugeordnet. Um hier mehr Transparenz zu schaffen, wurden die Ziele in ein weiteres Zielsystem überführt (siehe Abb. 2). Es gelten folgende Zusammenhänge:

- Intuitivität ist eines der zentralen Ziele bei der Gestaltung von IT-Systemen. Ist dieses Ziel erreicht, so werden Erlernbarkeit und Benutzbarkeit günstig beeinflusst. Die Verständlichkeit der Dokumentation beeinflusst die Erlernbarkeit.

- Die Fehlerfreiheit eines Informationssystems ist wünschenswert. Voraussetzung dafür sind die Testbarkeit und in der Regel auch ein hoher Entwicklungsstand (Ausgereiftheit). 


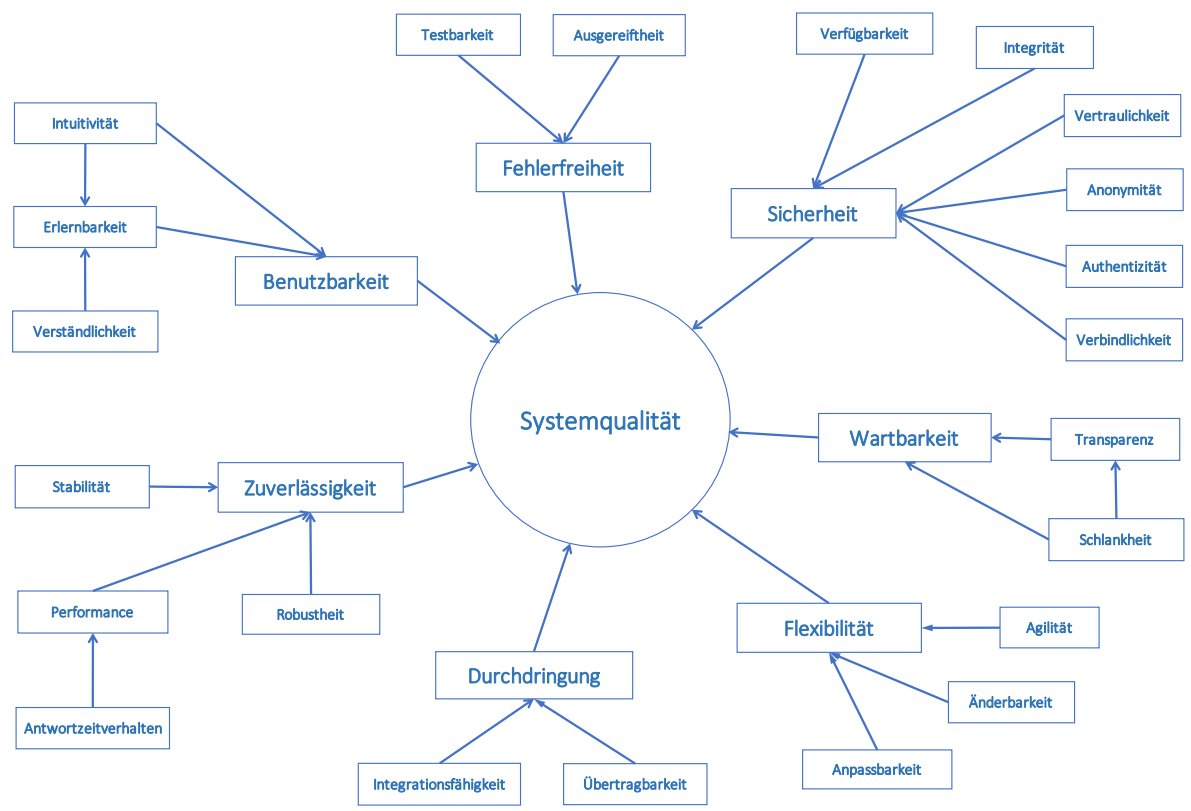

Abb. 2 Zielsystem von Teilzielen der Systemqualität

- Die wichtigsten Teilziele in Bezug auf Sicherheit von Informationssystemen sind Integrität, Verfügbarkeit, Vertraulichkeit, Anonymität, Authentizität und Verbindlichkeit (Heinrich et al. 2014, S. 211 f.).

- Die Schlankheit eines Systems wirkt sich auf die Transparenz und die Wartbarkeit eines Systems aus. Je schlanker ein System, desto einfacher ist seine Funktionsweise zu verstehen und eine Wartung durchzuführen.

- Eine hohe Flexibilität wird oft als Ziel gefordert. Anpassbarkeit, Agilität und Änderbarkeit beeinflussen Flexibilität.

- Eine hohe Durchdringung ist ebenfalls Teilziel der Systemqualität. Fördernd dafür sind eine hohe Integrationsfähigkeit der Teilsysteme sowie eine gute Übertragbarkeit.

- Stabilität, Robustheit und Performance beeinflussen die Zuverlässigkeit eines Systems. Das Antwortzeitverhalten ist dabei ein wesentlicher Aspekt von Performance.

Informationsqualität ist heute gut erforscht. Sie geht Hand in Hand mit der Qualität der Daten, die den Informationen zu Grunde liegen. Im Folgenden sind typische Qualitätsmerkmale aufgelistet, die für die Zielbildung herangezogen werden können (Heinrich et al. 2014, S. 288): Relevanz und Nutzen, Richtigkeit, Vollständigkeit, Aktualität, Genauigkeit, Konsistenz, Verständlichkeit und Verfügbarkeit.

Ebenfalls gut erforscht ist die Servicequalität. Das wohl wichtigste Standardwerk dazu ist die IT Infrastructure Library (ITIL). Sie ist eine Sammlung von Best Practices in einer Reihe von Publikationen zur Umsetzung eines IT-Service-Managements und gilt inzwischen als internationaler De-facto-Standard im Bereich IT- 
Geschäftsprozesse. In Service Level Agreements (SLA) werden die Qualitätskriterien definiert und zwischen dem Serviceanbieter und -nehmer vereinbart (vgl. dazu die Vorschlags- und Abstimmungsphase in einem Phasenmodell zur Entwicklung von SLA von Heinrich und Riedl 2003).

\section{Empirische Untersuchung}

Um die praktische Bedeutung wichtiger Formalziele empirisch zu erheben, wurden stichprobenartig IT-Verantwortliche unterschiedlichster Unternehmen zu 21 ausgewählten Formalzielen befragt. Zielgruppe der Befragung waren Personen, die ITVerantwortung in einem Unternehmen tragen. Die Befragung wurde im Herbst 2015 mit einem Online-Fragebogen durchgeführt, der per Email in Umlauf gebracht wurde.

\subsection{Fragebogendesign}

Die vorliegende Befragung hat explorativen Charakter. Im Hauptteil wurden folgende fünf Fragen zu jedem Ziel gestellt (jedes Ziel war mit einer Definition versehen, damit alle Befragten unter einem Ziel das gleiche Phänomen verstehen, vgl. Anhang):

1. Wird dieses Ziel in Ihrem Unternehmen verfolgt [ja, nein]?

2. Wenn ja, messen Sie dieses Ziel [ja, nein]?

3. Wenn ja, wie messen Sie dieses Ziel [Freitext]?

4. Wie wichtig ist dieses Ziel aus heutiger Sicht [1 = nicht wichtig, 2 = wenig wichtig, 3 = wichtig, 4 = sehr wichtig, $5=$ äußerst wichtig]?

5. Wie wichtig glauben Sie, wird dieses Ziel in fünf Jahren sein [Skala wie bei Frage (4)]?

\subsection{Demografische Daten}

An der Befragung haben 30 IT-Verantwortliche aus österreichischen Unternehmen verschiedenster Größen teilgenommen (siehe Abb. 3).

Die meisten Befragten sind IT-Leiter oder Geschäftsführer, gefolgt von IT-Managern und Systemadministratoren. Auch drei Entwickler haben an der Befragung teilgenommen, sowie eine Produktmanagerin aus der IT-Branche. Fünf Teilnehmer machten keine konkreten Angaben zur Position (vermutlich um die ohnehin von den Studienautoren zugesicherte Anonymität zu wahren).

Die Unternehmen kommen aus unterschiedlichsten Branchen. Die Teilnehmer sind zwischen 22 und 53 Jahre alt. Die überwiegende Mehrheit der Teilnehmer hat einen Hochschulabschluss. Bei sieben IT-Verantwortlichen ist das Abitur (in Österreich die Matura) die höchste abgeschlossene Ausbildung. Drei Teilnehmer verfügten über kein Abitur. 
Abb. 3 Unternehmensgröße $(\mathrm{N}=30)$

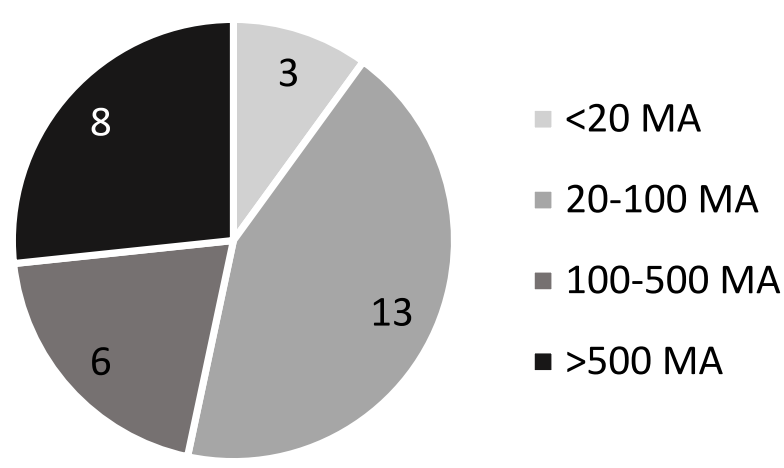

Fast alle Befragten mit Hochschulabschluss haben ein facheinschlägiges Studium absolviert. Es wurden Studien der Informatik, der Wirtschaftswissenschaften und kombinierte Studien (Technik und Betriebswirtschaft) genannt.

\subsection{Beurteilung der Zielverfolgung}

Alle Befragten geben an, mindestens 8 der 21 Ziele im Unternehmen zu verfolgen (siehe Abb. 4). Auf der X-Achse in Abb. 4 sind die 30 befragten Unternehmen gruppiert nach Größe aufgetragen, auf der y-Achse ist die Anzahl an Zielen angegeben. Etwa die Hälfte der Befragten geben an, mehr als zwei Drittel der vorgelegten Ziele in ihrem Unternehmen zu verfolgen. Anders sieht es bei der Messung der Ziele aus. Keiner der Befragten kann bei allen verfolgten Zielen bestätigen, dass diese auch gemessen werden (siehe Abb. 4). Nur in drei Unternehmen werden mehr als die Hälfte der verfolgten Ziele auch gemessen. Elf Befragte geben an, rund ein Drittel der verfolgten Ziele zu messen. Dazu ist jeweils auch angegeben, wie die Messung erfolgt. In sieben Unternehmen werden nur einzelne Ziele gemessen, jedoch weniger als $15 \%$ der verfolgten Ziele. Neun Befragte geben an, dass im Unternehmen keines der vorgeschlagenen Ziele gemessen wird bzw. werden keine Angaben zur Messung gemacht.

Verfolgte Ziele auch zu messen ist gemäß der vorliegenden Stichprobe eher in Großunternehmen verbreitet als in Kleinen und Mittleren Unternehmen (KMU). So geben sechs der acht IT-Verantwortlichen aus Großunternehmen an, mehr als $30 \%$ der verfolgten Ziele zu messen, während das nur in zwei der 22 restlichen Unternehmen der Fall ist (siehe Tab. 1). In keinem der Unternehmen mit weniger als 100 Beschäftigten werden laut den Verantwortlichen mehr als $50 \%$ der verfolgten Ziele gemessen. Aus den Antworten ist nicht ersichtlich, warum Ziele verfolgt, aber nicht gemessen werden. Gründe dafür könnten fehlendes Wissen oder mangelnde Datenqualität sein. Es ist auch möglich, dass die Verantwortlichen dafür schlichtweg keine Notwendigkeit sehen oder Zeit und Ressourcen fehlen, sich mit der Zielmessung näher zu befassen.

Elf der 30 Befragten geben an, sich im Rahmen der IT-Planung auch überlegt zu haben, welche Abhängigkeiten zwischen den Zielen bestehen, und können das mit einem Beispiel belegen (siehe Abb. 4). Für eine systematische Zielplanung ist 


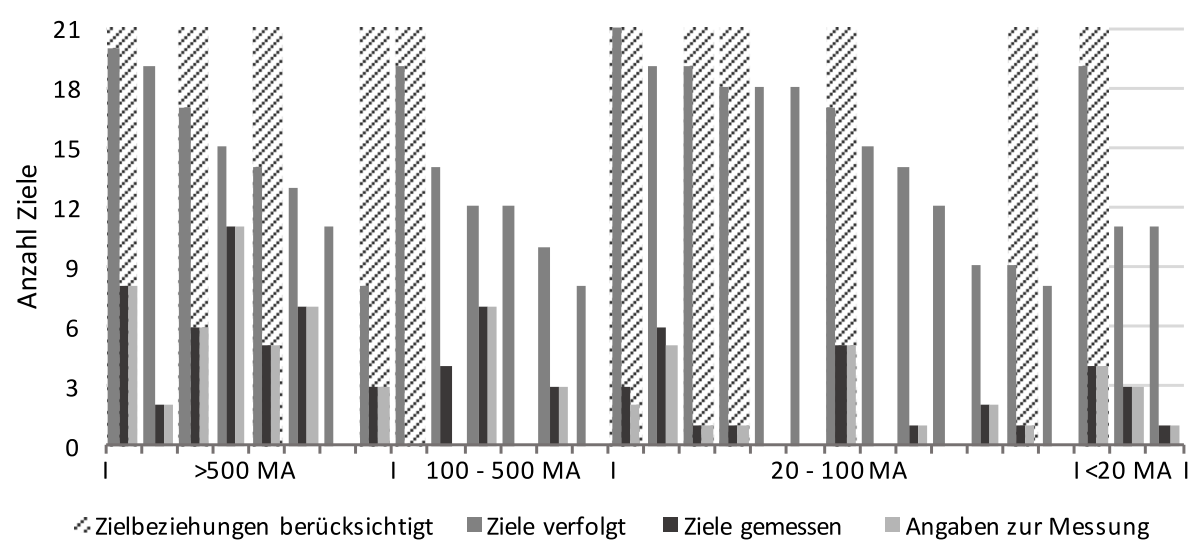

Abb. 4 Zielverfolgung je Unternehmen $(\mathrm{N}=30)$

das Berücksichtigen von Zielbeziehungen wesentlich. Bei allen anderen Unternehmen erfolgt die Zielverfolgung unabhängig von der Beachtung von Zielbeziehungen. Dass die Berücksichtigung von Zielbeziehungen abhängig von der Unternehmensgröße ist, kann anhand der vorliegenden Stichprobe nicht nachgewiesen werden. Es gibt in dieser Stichprobe sowohl Groß- als auch Kleinunternehmen, in denen Zielbeziehungen berücksichtigt als auch nicht berücksichtigt werden (siehe Tab. 2).

Eine Auswertung nach Zielen ergibt, dass Verfügbarkeit, Nutzerzufriedenheit, Zuverlässigkeit und Sicherheit von mehr als $90 \%$ der Unternehmen aus der Stichprobe verfolgt werden (siehe Abb. 5). Mehr als $80 \%$ der Unternehmen verfolgen außerdem Wartbarkeit und Wirksamkeit als Ziel. Auch Fehlerfreiheit und Stabilität wird von mehr als drei Viertel der befragten Unternehmen als Ziel verfolgt. Am seltensten, aber dennoch von $40 \%$ der Unternehmen, wird die Informationsquantität als Ziel verfolgt. Danach folgen Nachhaltigkeit, Entscheidungsunterstützung und Durchdringung als am wenigsten oft verfolgte Ziele.

Die Ziele Verfügbarkeit, Sicherheit und Nutzerzufriedenheit werden nicht nur von fast allen Unternehmen der Stichprobe verfolgt, sondern auch am häufigsten gemessen. Etwa ein Drittel der Unternehmen, die diese Ziele verfolgen, messen diese auch. Ebenso häufig gemessen wird das Antwortzeitverhalten (vermutlich deshalb, weil es durch den Einsatz technischer Verfahren relativ einfach messbar ist). Auch Wirtschaftlichkeit, Fehlerfreiheit, Zuverlässigkeit und Stabilität werden von mehreren Unternehmen gemessen. Von keinem der Unternehmen werden Anpassbarkeit und Nachhaltigkeit gemessen (vermutlich aufgrund der Messproblematik, vgl. dazu Abschn. „Diskussion der Ergebnisse“ dieses Beitrags).

\subsection{Beurteilung der Wichtigkeit}

Die Angaben zur Wichtigkeit der Ziele sind ähnlich zu den Angaben zur Zielverfolgung. Je häufiger ein Ziel verfolgt wird, desto wichtiger wird es eingeschätzt. Zuverlässigkeit und Sicherheit werden dabei als die wichtigsten Ziele angegeben (siehe Abb. 6). Deutlich an Bedeutung gewinnen werden Entscheidungsunterstüt- 
Tab. 1 Anteil der gemessenen Ziele an den verfolgten Zielen nach Unternehmensgröße (N = 30)

\begin{tabular}{llllll}
\hline & \multicolumn{2}{l}{ Anzahl Beschäftigte } & & \\
Ziele gemessen in \% & $<20$ & $20-100$ & $101-500$ & $>500$ & Gesamtergebnis \\
\hline Kein Ziel gemessen & 1 & 5 & 3 & 1 & 9 \\
1 bis 15 & 2 & 2 & 2 & 1 & 7 \\
16 bis 30 & & 1 & & 4 & 6 \\
31 bis 50 & 3 & 13 & 6 & 2 & 3 \\
Über 50 gemessen & & & & 8 & 30 \\
Gesamtergebnis & & & & & 5 \\
\hline
\end{tabular}

Tab. 2 Zielbeziehungen berücksichtigt nach Unternehmensgröße $(\mathrm{N}=30)$

\begin{tabular}{llllll}
\hline & \multicolumn{2}{l}{ Anzahl Beschäftigte } & & \\
$\begin{array}{l}\text { Zielbeziehungen berücksich- } \\
\text { tigt }\end{array}$ & $<20$ & $20-100$ & $101-500$ & $>500$ & Gesamtergebnis \\
\hline Ja & 1 & 5 & 1 & 4 & 11 \\
Nein & 2 & 8 & 5 & 4 & 19 \\
Gesamtergebnis & 3 & 13 & 6 & 8 & 30 \\
\hline
\end{tabular}

zung und Nachhaltigkeit als Ziele von Informationssystemen. Beide werden heute jedoch als unterdurchschnittlich wichtig eingeschätzt. Nach Angaben der Befragten wird die Wirtschaftlichkeit gleichfalls deutlich an Bedeutung gewinnen. Auch Teilziele der Systemqualität wie Schlankheit, Wartbarkeit, Anpassbarkeit und Integrationsfähigkeit werden nach Ansicht der Befragten überdurchschnittlich stark an Bedeutung gewinnen, gleiches gilt für die Informationsqualität.

\section{Diskussion der Ergebnisse}

Auf der Basis einer Literaturanalyse wurden in dieser Arbeit wichtige Formalziele von Informationssystemen identifiziert und in ein Zielsystem überführt. In einer auf dieser Analyse aufbauenden Online-Befragung wurden IT-Verantwortliche gebeten, Auskunft zu ausgewählten Zielen zu geben. Überraschend ist, dass Wirtschaftlichkeit aus heutiger Sicht nur als durchschnittlich wichtig eingestuft wird. Nur in jedem vierten Unternehmen der Stichprobe wird die Wirtschaftlichkeit von Informationssystemen gemessen. Laut Angaben der Befragten wird die Wirtschaftlichkeit aber nicht fortlaufend während dem Betrieb der Informationsinfrastruktur gemessen, sondern nur einmalig bei Planungstätigkeiten überprüft, beispielsweise im Zuge einer Investitionsrechnung. Nur ein Befragter gibt an, den Anteil der ITKosten am Umsatz als Key Performance Indicator (KPI) für die Wirtschaftlichkeit der Informationssysteme heranzuziehen.

Wenig überraschend ist, dass Sicherheit, Zuverlässigkeit und Verfügbarkeit als die wichtigsten Ziele angegeben werden. Ein Ausfall von Informationssystemen kann für die meisten Unternehmen heute schwerwiegende Folgen haben. Nur in wenigen 


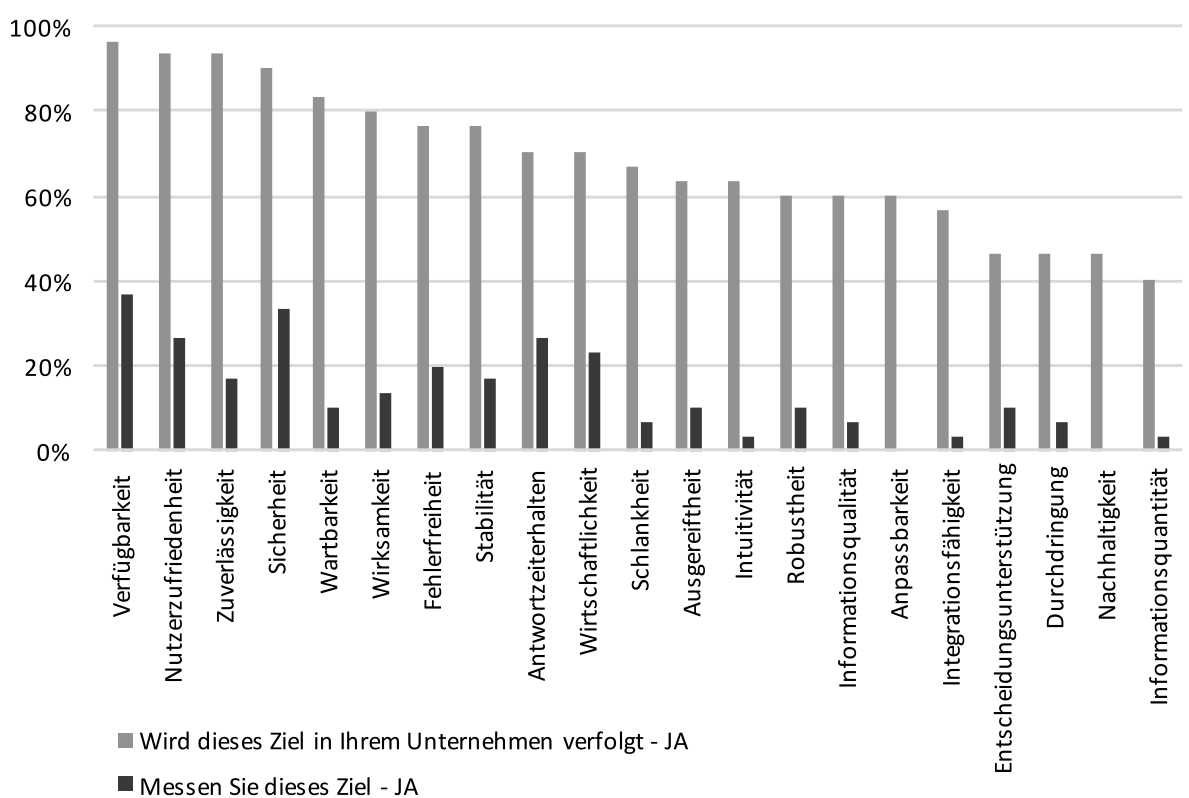

Abb. 5 Zielverfolgung in der Praxis $(\mathrm{N}=30)$

Branchen bzw. Unternehmen ist die IT-Durchdringung heutzutage so gering, dass ein Ausfall der IT über mehrere Tage ohne gravierende Schäden verkraftbar ist.

Die Ergebnisse der Befragung zeigen weiter, dass nur in den wenigsten Unternehmen eine konkrete Zielplanung gemacht wird. Verantwortliche geben zwar an, die meisten der genannten Ziele für Informationssysteme im Unternehmen zu verfolgen und schätzen deren Bedeutung auch durchwegs als hoch ein, dennoch führen sie aber größtenteils keine Zielmessung durch. Eine Mehrheit der IT-Verantwortlichen gibt zudem an, dass sie sich bei der Zielplanung keine Gedanken über Zielbeziehungen machen. Dieser Umstand ist problematisch, da zumindest einige Ziele von Informationssystemen in einem konfliktären, nicht-linearen Zusammenhang stehen (siehe z. B. ein Beispiel in Heinrich et al. 2011, S. 249 f.).

In einer Gesamtschau lassen die Ergebnisse der vorliegenden Studie den Schluss $\mathrm{zu}$, dass es in vielen Unternehmen keine systematische Zielplanung gibt. Ein Grund dafür könnte fehlendes Wissen der Verantwortlichen sein. Es kann offensichtlich nicht davon ausgegangen werden, dass IT-Verantwortliche umfangreiches Wissen zur Planung von Zielen von Informationssystemen haben. Andere, nicht notwendigerweise widersprüchliche Erklärungen sind, dass IT-Verantwortliche für eine systematische Zielplanung schlichtweg (noch) keine Notwendigkeit sehen oder dermaßen mit operativen Aspekten des IT-Managements befasst sind, so dass für strategische Aufgaben wie die Zielplanung keine oder nur unzureichend Zeit und/oder personelle Ressourcen zur Verfügung stehen. 
5

4

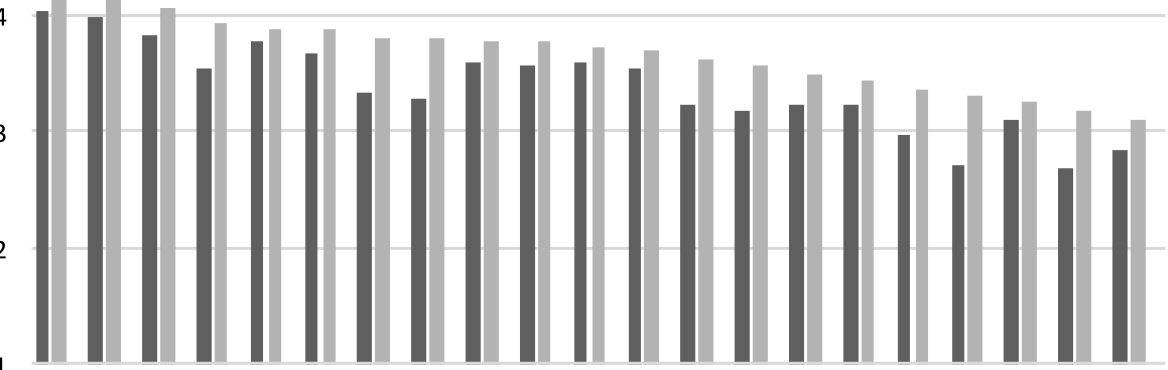

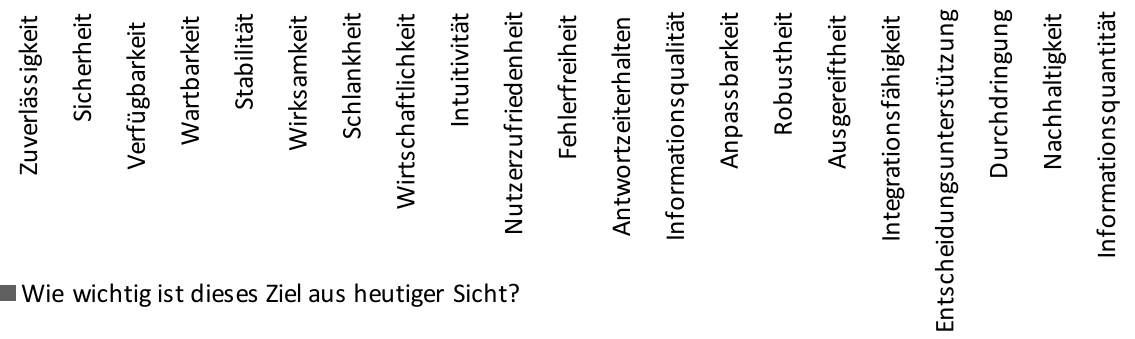

Wie wichtig glauben Sie, wird dieses Ziel in 5 Jahren sein?

Mittelwerte: 1 = nicht wichtig, 2 = wenig wichtig, 3 = wichtig, 4 = sehr wichtig, 5 = äusserst wichtig

Abb. 6 Einschätzung der Wichtigkeit $(\mathrm{N}=30)$

\section{Handlungsempfehlungen und Ausblick}

Die in den Abb. 1 und Abb. 2 dargestellten Ziele inklusive deren Abhängigkeiten können in Unternehmen bei der strategischen Zielplanung als konzeptionelle Grundlage verwendet werden. Darauf aufbauend können IT-Verantwortliche unmittelbar Zielinhalte ableiten sowie in weiterer Folge Ziele priorisieren, Beziehungen zwischen den Zielen thematisieren und weitere Aspekte einer systematischen Zielplanung diskutieren (z. B. Zeitpläne zur Zielmessung entwickeln und Zielverantwortliche festlegen). Mögliche KPI zur Messung der Zielerreichung können anschließend selbst entwickelt oder gezielt in der Fachliteratur recherchiert werden (vgl. dazu z. B. einschlägige Lehrbücher des Informationsmanagements und IT-Controllings). Sofern Unternehmen die Zielerreichung der bedeutsamsten strategischen Formalziele (vgl. Abb. 1) nicht messen, sollte zumindest eine Einschätzung der Zielerreichung durch IT-Verantwortliche geschehen. Dadurch wird der Istzustand zumindest rudimentär erhoben. Darauf aufbauend können Soll-Zielwerte definiert werden. Eine solche Vorgehensweise ist nach Ansicht der Verfasser dieses Beitrags einer gänzlich fehlenden Auseinandersetzung mit Zielen von Informationssystemen vorzuziehen und zudem kann sie den Einstieg in eine zunehmende organisationale Messprofessionalisierung im IT-Bereich darstellen. Grundsätzlich ist in Unternehmen darauf zu achten, Angemessenheit und Wirksamkeit eines entwickelten Zielsystems zu überprüfen, bevor es dauerhaft zum Einsatz kommt. 
Das in diesem Beitrag entwickelte Zielsystem (Abb. 1 und daraus abgeleitet weiter präzisierend Abb. 2) kann neben dem Einsatz in der Praxis auch als Grundlage für die weitere Zielforschung dienen. Erstens sollte es in Fachkreisen diskutiert werden, um beispielsweise festzustellen, ob die Liste der Ziele reduziert oder erweitert werden sollte. Zweitens sollten die angenommenen Abhängigkeiten empirisch überprüft werden. Drittens sollten sich weiterführende Arbeiten damit befassen, konkrete Möglichkeiten zur Messung der einzelnen Ziele aufzuzeigen. Idealtypisch sollte die Messung eines Ziels mit möglichst wenig Aufwand und geringen Kosten verbunden sein. Insbesondere wäre dieser dritte Punkt ein weiterer wichtiger Schritt, um eine systematische Zielplanung im Bereich der Informationssysteme in der Praxis voranzutreiben, und zwar deshalb, weil die Messproblematik (eine Einführung in die Grundlagen des Messens findet sich z. B. bei Heidenreich 1999) eine wesentliche Erklärung dafür ist, warum in vielen Unternehmen heute nach wie vor keine systematische Zielplanung etabliert wurde, obwohl eine solche seit den 1980er-Jahren in der Fachliteratur gefordert wird.

\section{Anhang}

Die folgenden Formalziele wurden in der Online-Befragung untersucht. Die Definitionen stammen aus Heinrich et al. (2004, 1, 2, 4, 5, 6, 9, 12, 13, 14, 16, 17, 18, 19), Heinrich et al. (2011, 15, 20, 21), Heinrich et al. (2014, 7) sowie DeLone und McLean (2003, 3, Übersetzung aus dem Englischen durch die Verfasser); eigene Definition $(8,10,11)$.

1. Anpassbarkeit

Anpassbarkeit beschreibt die Eigenschaft eines Systems, auf qualitative und quantitative Änderungen der Anforderungen ohne grundlegende Veränderung des Systems reagieren zu können. Dabei wird angenommen, dass die notwendigen Veränderungen vorausgeplant sind.

2. Antwortzeitverhalten

Die Antwortzeit gibt Auskunft über die Dauer von Beendigung einer Benutzereingabe bis das erste Zeichen der Antwort dazu vorliegt. Ein gutes Antwortzeitverhalten ist eine kurze und stabile Antwortzeit für eine Menge gleicher o. ä. Transaktionen über die Zeit.

3. Ausgereiftheit

Die Ausgereiftheit eines Systems beschreibt einen hohen Entwicklungsstand.

4. Durchdringung

Die Durchdringung beschreibt das Ausmaß, in dem die Informationsinfrastruktur betriebliche Aufgaben unterstützt.

5. Entscheidungsunterstützung

Entscheidungsunterstützung beschreibt die Fähigkeit eines Systems, BenutzerInnen im Entscheidungsprozess zu unterstützen. Dieser gliedert sich in die Phasen Problemerkennung, Informationsgewinnung, Alternativenentwurf und Auswahl der optimalen Alternative. 


\section{Fehlerfreiheit}

Fehlerfreiheit bedeutet, dass es keine negativen Abweichungen vom geplanten oder in einer Spezifikation festgelegten Zustand gibt.

7. Informationsqualität

Informationsqualität drückt aus, in welchem Ausmaß eine Information geeignet ist, den Informationsbedarf zu decken.

8. Informationsquantität

Informationsquantität beschreibt die Menge von Informationen.

9. Integrationsfähigkeit

Die Integrationsfähigkeit beschreibt die Fähigkeit eines Systems, mit anderen Systemen so zu interagieren, dass ein Betrachter des Systems den Eindruck hat, es handle sich nicht um ein einzelnes System, sondern um den Teil eines umfassenden Gesamtsystems.

10. Intuitivität

Intuitivität ist die Eigenschaft eines Systems, die es BenutzerInnen ermöglicht, es ohne Schulung nutzen zu können.

11. Nachhaltigkeit

Nachhaltigkeit beschreibt die Eigenschaft eines Systems langfristig ökologisch, ökonomisch und sozial verträglich eingesetzt werden zu können.

12. Nutzerzufriedenheit

Nutzerzufriedenheit (oder Benutzerzufriedenheit) ist eine spezifische Form der Arbeitszufriedenheit, die auf Personen orientiert ist, welche Informationssysteme benutzen.

\section{Robustheit}

Robustheit ist die Fähigkeit eines Systems, auftretende Fehler ohne Gefährdung seiner Funktionalität tolerieren zu können oder - wenn dies nicht mehr möglich ist - Fehlreaktionen nur in einem vernünftigen Verhältnis zum auslösenden Fehler zu zeigen.

\section{Schlankheit}

In Anlehnung an Lean Management ist die Schlankheit eines Informationssystems die Fähigkeit, den folgenden Prinzipien zu entsprechen: Ganzheitliche Orientierung, Reduzierung von Komplexität, intensive Kommunikation und Rückkoppelung zwischen Aufgabenträger und Prozessabschnitten sowie Schaffung langfristiger wechselseitiger Verpflichtungen.

15. Sicherheit

Sicherheit beschreibt die Eigenschaft eines Systems, Bedrohungen so begegnen zu können, dass Schäden vermieden werden.

16. Stabilität

Stabilität ist die Fähigkeit eines Systems, auf gleichartige o. ä. Impulse immer gleich zu reagieren.

17. Verfügbarkeit

Verfügbarkeit ist die Fähigkeit eines Systems, seine konstruktionsbedingten Funktionen erfüllen zu können. Verfügbarkeit ist damit gleichbedeutend mit der Wahrscheinlichkeit, das System zu einem bestimmten Zeitpunkt in einem funktionsfähigen Zustand anzutreffen. 


\section{Wartbarkeit}

Wartbarkeit ist die Eigenschaft eines Systems an veränderte Anforderungen anpassbar zu sein.

19. Wirksamkeit

Wirksamkeit ist die Eigenschaft eines Systems, die geplanten Funktionen und Leistungen zu erbringen.

20. Wirtschaftlichkeit

Wirtschaftlichkeit beschreibt den mit Kosten bewerteten Verbrauch an Gütern und Dienstleistungen, die für die Systemnutzung eingesetzt werden, im Verhältnis zum wertmäßigen Ergebnis der Systemnutzung.

\section{Zuverlässigkeit}

Zuverlässigkeit beschreibt die Wahrscheinlichkeit der Einhaltung aller anderen Formalziele der Systemnutzung.

Open Access. This article is distributed under the terms of the Creative Commons Attribution 4.0 International License (http://creativecommons.org/licenses/by/4.0/), which permits unrestricted use, distribution, and reproduction in any medium, provided you give appropriate credit to the original author(s) and the source, provide a link to the Creative Commons license, and indicate if changes were made.

\section{Literatur}

DeLone WH, McLean ER (2003) The DeLone and McLean Model of Information Systems Success: A Ten-Year Update. J Manag Inf Syst 19(4):9-30

Heidenreich K (1999) Grundbegriffe der Mess- und Testtheorie. In: Roth E, Holling H (Hrsg) Sozialwissenschaftliche Methoden, 5. Aufl. Oldenbourg, München, S 342-374

Heinrich LJ, Sterrer G (1987) Ziele von Informationssystemen: Ergebnisse einer empirische Studie. Inf Manag 2(1):48-53

Heinrich LJ, Heinzl A, Riedl R (2011) Wirtschaftsinformatik: Einführung und Grundlegung, 4. Aufl. Oldenbourg, München

Heinrich LJ, Heinzl A, Roithmayr F (2004) Wirtschaftsinformatik-Lexikon, 7. Aufl. Oldenbourg, München

Heinrich LJ, Riedl R, Stelzer D (2014) Informationsmanagement; Grundlagen, Aufgaben, Methoden, 11. Aufl. De Gruyter, Berlin

Heinrich LJ, Riedl R (2003) Phasenmodell zur Entwicklung von Serviceebenen-Vereinbarungen. HMD Prax Wirtschaftsinformatik 40(231):88-96

Krcmar H (2010) Informationsmanagement, 5. Aufl. Springer, Heidelberg

Mertens P, Modendorf F, König W, Picot A, Schumann M, Hess T (2012) Grundzüge der Wirtschaftsinformatik, 11. Aufl. Springer/Gabler, Berlin

Roithmayr F (1988) Controlling von Informations- und Kommunikationssystemen. Oldenbourg, München

Teubner RA (2013) Informationssystem-Strategie: Theorie, Praxis und Herausforderungen an die Forschung. WIRTSCHAFTSINFORMATIK 55(4):239-255 